\title{
Limits on the Number of Function Evaluations Required by Certain High-Dimensional Integration Rules of Hypercubic Symmetry
}

\author{
By J. N. Lyness
}

Abstract. We consider an $n$-dimensional integration rule $R_{t}{ }^{(n)}$ of degree $2 t-1$ and of hypercubic symmetry. We derive theorems which set a lower bound in terms of $n$ and $t$ on the number of function evaluations such a rule requires. These results apply to spaces of integration which have hypercubic symmetry. In certain cases this bound is very close to the number of points required by a known rule.

1. Introduction. In previous papers (Lyness [1], [2], [3]), referred to as Parts I, II, and III, respectively, we considered in detail a certain type of $n$-dimensional symmetric integration rule $R^{(n)}$ of hypercubic symmetry suitable for integration over an $n$-dimensional hypercube. The rule $R^{(n)}$ may be expressed in terms of basic $n$-dimensional rules $R_{i}{ }^{(n)}$ defined in Part I,

$$
R^{(n)}=\sum \xi_{i} Q_{i}^{(n)}, \quad \xi_{i} \neq 0 .
$$

We express this relation by stating that $R^{(n)}$ includes the basic rule $R_{i}{ }^{(n)}$. In Part III we constructed high-dimensional rules of degree $2 t+1$ which required as few function evaluations as we could conveniently arrange.

The construction of economical high-dimensional integration rules of particular degree is an intricate problem. A common procedure is to write down a composite rule $R^{(n)}$ which contains sufficient undetermined parameters and then to write down the nonlinear equations*

$$
R^{(n)} f=I^{(n)} f,
$$

where $f$ is in turn one of a set of polynomials (the elements of $\Phi_{t+1}^{(n)}$ of Lyness [2, Section 4]). These simultaneous nonlinear equations are difficult to solve and it may easily happen that the form of $R^{(n)}$ is such that there are no solutions to these equations. In this paper we establish certain theorems which give information about the form of $R^{(n)}$ and so reduce the likelihood of fruitless attempts to solve sets of simultaneous nonlinear equations which have no solution. We illustrate the type of theorem in the following rather trivial example.

We might search for a two-dimensional integration rule of degree 5 by insisting on using as evaluation points only points for which $\left|x_{1}\right|=\left|x_{2}\right|$. In the notation of Lyness [1], this means a rule of the form

$$
R^{(2)}=\sum \xi_{i} \Re\left(\alpha_{i}, \alpha_{i}\right),
$$

where $\xi_{i}$ are weighting factors.

Received November 10, 1964. This work was supported in part by U. S. Air Force Grant No. 685-64 to the University of New South Wales.

${ }^{*} R^{(n)} f$ is the result of applying rule $R^{(n)}$ to the function $f . I^{(n)} f$ is the exact $n$-dimensional integral of this function over the unit hypercube. 
We are then led to the four equations:

$$
R^{(2)} f=I^{(2)} f
$$

with $f$ being in turn the polynomials $1, x_{1}{ }^{2}, x_{1}{ }^{4}$, and $x_{1}{ }^{2} x_{2}{ }^{2}$. These equations are in fact insoluble. This can be seen if we calculate $R\left(\alpha_{i}, \alpha_{i}\right)\left(x_{1}{ }^{2}-x_{2}{ }^{2}\right) x_{1}{ }^{2}$. This basic rule evaluates any function only at points where $x_{1}^{2}=x_{2}^{2}$, that is, at points where the function $\left(x_{1}{ }^{2}-x_{2}{ }^{2}\right) x_{1}{ }^{2}$ is zero. Thus

$$
R\left(\alpha_{i}, \alpha_{i}\right)\left(x_{1}^{2}-x_{2}^{2}\right) x_{1}^{2}=0,
$$

and so

$$
R^{(2)}\left(x_{1}^{2}-x_{2}^{2}\right) x_{1}^{2}=\sum \xi_{i} \mathcal{R}\left(\alpha_{i}, \alpha_{i}\right)\left(x_{1}^{2}-x_{2}^{2}\right) x_{1}^{2}=0 .
$$

But

$$
\begin{aligned}
I^{(2)}\left(x_{1}{ }^{2}-x_{2}{ }^{2}\right) x_{1}{ }^{2} & =I^{(2)} x_{1}^{4}-I^{(2)} x_{1}^{2} x_{2}^{2} \\
& =\frac{1}{5}-\frac{1}{9} \neq 0 .
\end{aligned}
$$

Thus

$$
R^{(2)} f \neq I^{(2)} f
$$

for at least one function $f$ of degree 4 , and so $R^{(2)}$ cannot be of degree 5 if it is of form (1.3) above.

This result could be stated in the form that all two-dimensional rules of degree 5 must include a basic rule $R(\alpha, \beta)$ with $\alpha \neq \beta$. It also shows that the number of points required by a two-dimensional rule of degree 5 of hypercubic symmetry is equal to or greater than the smallest possible $\nu(R(\alpha, \beta))$ which is 4 .

In this paper we establish several theorems which place restrictions of the above type on the form of an $n$-dimensional rule $R_{t+1}^{(n)}$ of hypercubic symmetry and of degree $2 t+1$. To do this we classify into sets the basic rules $R^{(n)}$ and show that $R_{t+1}^{(n)}$ must include members of certain sets or combinations of sets. The proofs of these theorems follow the same lines as the proof of the result stated above, but are of course more complicated.

2. Sets and Classes of Basic Rules. An $n$-dimensional basic rule may be written in the form

$$
\mathbb{R}^{(n)}=R(0)^{n_{0}} * R\left(\alpha_{1}\right)^{n_{1}} * R\left(\alpha_{2}\right)^{n_{2}} * \cdots * R\left(\alpha_{\varepsilon}\right)^{n_{*}},
$$

where the $\alpha_{i}$ are $s$ distinct nonzero coordinates and the $n_{i}$ are positive integers arranged so that

$$
n_{1} \geqq n_{2} \geqq \cdots \geqq n_{\mathrm{s}}>0 .
$$

Different rules $Q^{(n)}$ which are defined by the same set of integers $n_{0}, n_{1}, \cdots, n_{s}$ (but which may have different coordinates $\alpha_{i}$ ) are defined as belonging to the same class. Basic rules belonging to the same class require the same number of function evaluations $\nu\left(R^{(n)}\right)$.

We define three sets of the rules $R^{(n)}$ given by (2.1) and (2.2) above. These are

$$
\mathfrak{R}^{(n)} \in \rho_{i, 1}^{(n)} \text { if } n-n_{0} \geqq i \text { and } s \geqq 1 \text {, }
$$




$$
\begin{aligned}
& \mathbb{R}^{(n)} \in \sigma_{i, 1}^{(n)} \quad \text { if } \quad n-n_{0} \geqq i \text { and } s=1 \text {, } \\
& \mathcal{R}^{(n)} \in \rho_{i, 2}^{(n)} \text { if } n-n_{0} \geqq i \text { and } s \geqq 2 \text {. }
\end{aligned}
$$

We define $\bar{\nu}(\sigma)$, the minimal number of points of a set $\sigma$, by the equation

$$
\bar{\nu}(\sigma)=\min _{R \in \sigma} \nu(R)
$$

and $R$ is a minimal rule of $a$ set $\sigma$ if and only if

$$
R \in \sigma \text { and } \nu(R)=\bar{\nu}(\sigma) .
$$

Finally we define

$$
\bar{\nu}\left(\sigma, \sigma^{\prime}\right)=\min _{R \in \sigma ; R^{\prime} \in \sigma^{\prime}}\left(\nu(R)+\nu\left(R^{\prime}\right)\right),
$$

where $R$ and $R^{\prime}$ are in different classes.

The values of $\bar{\nu}(\sigma)$ may be calculated using the formulas given in Section 5 of Part I. We find in particular $\bar{\nu}\left(\sigma_{t-1,1}^{(n)}, \sigma_{t, 1}^{(n)}\right)$

$$
=\min \left(2^{t-1}\left(\begin{array}{c}
n \\
t-1
\end{array}\right)+2^{t}\left(\begin{array}{l}
n \\
t
\end{array}\right), 2^{t-1}\left(\begin{array}{c}
n \\
t-1
\end{array}\right)+2^{n}, 2^{n-1}\left(\begin{array}{c}
n \\
n-1
\end{array}\right)+2^{n}\right) .
$$

We also find

$$
\begin{aligned}
\bar{\nu}\left(\rho_{i, 1}^{(n)}\right) & =\bar{\nu}\left(\sigma_{i, 1}^{(n)}\right)=\min \left(2^{i}\left(\begin{array}{l}
n \\
i
\end{array}\right), 2^{n}\right) \\
& \leqq \frac{1}{i} \bar{\nu}\left(\rho_{i, 2}^{(n)}\right)=\frac{1}{i} \min \left(i 2^{i}\left(\begin{array}{l}
n \\
i
\end{array}\right), n 2^{n}\right) .
\end{aligned}
$$

These inequalities indicate that in constructing a rule $R$ we should avoid if it is possible the use of basic rules from the sets $\rho_{i, 2}^{(n)}$ as we may use up to $i-1$ different rules from the set $\sigma_{i, 1}^{(n)}$ instead at a lower cost in terms of number of points. We should also avoid as far as possible rules from sets $\rho_{i, 1}^{(n)}$ with large $i$. The theorems of the next section indicate how far it is possible to do this if the rule $R^{(n)}$ is to be of degree $2 t+1$.

3. Bounds on $\bar{\nu}\left(S_{t+1}^{(n)}\right)$. We define the set $S_{t+1}^{(n)}$ as the set of all $n$-dimensional integration rules of hypercubic symmetry of degree $2 t+1$. In this section we state $\dagger$ a set of theorems which together place a lower bound on $\bar{\nu}\left(\delta_{t+1}^{(n)}\right)$. For $n>t$, this bound is relatively close to the number of points required by known integration rules.

Theorem 3.1. If an n-dimensional rule $R^{(n)}$ is of the form

$$
R^{(n)}=(R(0))^{n-8} * R^{(s)}
$$

where $R^{(s)}$ is any s-dimensional composite rule, and

$$
f=\left(x_{1}^{2} x_{2}^{2} \cdots x_{s+1}^{2}\right) g\left(x_{1}, x_{2}, \cdots, x_{n}\right),
$$

$\dagger$ The proofs of these theorems and a wider discussion of the definitions are available in preprint form from the author. 
where $g$ is any polynomial, then

$$
R^{(n)} f=0 .
$$

Two rather simple corollaries of this theorem follow;

TheOREM 3.1A.

$$
(R(0))^{n-s} * R^{(s)} f=0 \text { when } f \in \phi_{t+1}^{m}, m>s+1 .
$$

Theorem 3.1B.

$$
E_{s}^{(n)}(0) R^{(s)} f=0 \text { when } f \in \phi_{t+1}^{m}, m>s+1 .
$$

The sets $\phi_{t+1}^{m}$ are defined and discussed in Section 4 of Part II.

TheOREM 3.2. If $f=\left(x_{1}{ }^{2}-x_{2}{ }^{2}\right) g\left(x_{1}, x_{2}, \cdots, x_{n}\right)$, where $g$ is any polynomial, then

$$
(R(\alpha))^{n} f=0 .
$$

Theorems 3.1, 3.1A, 3.1B, and 3.2 play a basic part in the proofs of the remaining theorems of this section.

TheOREM 3.3. An n-dimensional rule $R_{t+1}^{(n)}$ of degree $2 t+1$ includes a basic rule of the set $\rho_{t^{\prime}, 1}^{(n)}$, where $t^{\prime}=\min (n, t)$.

Before going on to the next theorem, we emphasize the importance of this theorem for $n>t$. Using only the results that

$$
I^{(n)} x_{1}^{2} x_{2}{ }^{2} \cdots x_{t^{\prime}}^{2} \neq 0
$$

and that the rule has hypercubic symmetry, we have shown that

$$
\bar{\nu}\left(\zeta_{t+1}^{(n)}\right) \geqq \bar{\nu}\left(\rho_{t, 1}^{(n)}\right)=\min \left(2^{t}\left(\begin{array}{l}
n \\
t
\end{array}\right), 2^{n}\right) .
$$

This number may be large. In Part III we obtained a rule $\bar{W}_{5}^{(15)}$ which requires 26,921 function evaluations. This rule satisfies

$$
\bar{W}_{5}^{(15)} f=I^{(15)} f
$$

when $f$ is any of the twelve elements of $\Phi_{4}{ }^{(15)}$. However, to satisfy this equation when $f$ is the element $x_{1}{ }^{2} x_{2}{ }^{2} x_{3}{ }^{2} x_{4}{ }^{2}$ requires 21,840 points. Although we cannot strictly state that particular points belong in any sense to particular functions, in this case it is correct to infer that, but for having to satisfy this one equation, we could satisfy the remaining eleven equations using less than 5,200 points. Thus, in this example $(n=15 ; t=4)$ we see that Theorem 3.3 gives a bound which is $80 \%$ of the lowest known value, and that this $80 \%$ of the points arise from having to satisfy one of the twelve defining equations. This bound is the one listed in Table 1 of Part II.

The remaining theorems are of the same type as this theorem. Their effect is to raise the lower bound on $\bar{\nu}\left(\mathcal{S}_{t+1}^{(n)}\right)$.

Theorem 3.4. An n-dimensional rule $R_{t+1}^{(n)}$ of degree $2 t+1, t \geqq 4$, includes a basic rule of the set $\rho_{t^{\prime}, 2}^{(n)}$, where $t^{\prime}=\min (n, t-2)$.

THEOREM 3.5. An n-dimensional rule $R_{t+1}^{(n)}$ of degree $2 t+1$ which includes no basic rule from the set $\rho_{t-1,2}^{(n)}$ either includes two rules of different classes from the set 
$\sigma_{t-1,1}^{(n)}$ or includes one or more rules of the class $R(0)^{n-8} * R(\alpha)^{s}$ if there exists an integer $s=t-1+k(n-t+1)>t$, where

$$
k=\frac{I^{(n)} x_{1}^{2} x_{2}^{2} \cdots x_{t}^{2}}{I^{(n)} x_{1}^{4} x_{2}^{2} \cdots x_{t-1}^{2}}
$$

The dependence of these theorems on the region of integration is relatively slight. Theorem 3.3 depends on

$$
I^{(n)} x_{1}^{2} x_{2}^{2} \cdots x_{t}^{2} \neq 0
$$

Theorem 3.4 depends on

$$
I^{(n)}\left(x_{1}^{2}-x_{2}^{2}\right) x_{1}^{4} x_{2}^{2} \cdots x_{t}^{2} \neq 0
$$

and Theorem 3.5 requires further

$$
I^{(n)} x_{1}^{4} x_{2}^{2} \cdots x_{t-1}^{2} \neq 0
$$

and the value of $k$ in Theorem 3.5 is the ratio of the first to the third of these. We are interested in regions of integration symmetric under the interchange of coordinates, so it follows that

$$
I^{(n)}\left(x_{1}^{2}-x_{2}^{2}\right) x_{1}^{4} x_{2}^{2} \cdots x_{t}^{2}=\frac{1}{2} I^{(n)}\left(x_{1}^{2}-x_{2}^{2}\right)^{2} x_{2}^{2} \cdots x_{t}{ }^{2} .
$$

Since the integrand in each case is non-negative definite, the above inequalities may be relied upon.

The value of $k$ does affect the number of points required. In the case of integration over a hypercube, we have

$$
k=\frac{5}{8} \text {. }
$$

In the case of integration over an infinitely extended region with weight function $\exp \left[-\sum x_{i}{ }^{2}\right]$ (see Stroud and Secrest [5] or Lyness [4]), we find

$$
k=\frac{1}{3} \text {. }
$$

4. A Lower Bound on $\bar{\nu}\left(\delta_{t+1}^{(n)}\right)$. Theorems $3.3,3.4$, and 3.5 may be used to set a bound on the number of points required by $R_{t+1}^{(n)}$. Considering the cases in which $n \geqq t \geqq 4$ only, we find that these theorems may be combined to yield

Theorem 4.1. An n-dimensional rule $R_{t+1}^{(n)}(n \geqq t \geqq 4)$ of degree $2 t+1$, either

(a) includes a basic rule of the set $\rho_{t, 2}^{(n)}$, or

(b) includes a basic rule of the set $\sigma_{t, 1}^{(n)}$, and one of the set $\rho_{t-1,2}^{(n)}$, or

(c) includes a basic rule of the set $\sigma_{t, 1}^{(n)}$, one of the set $\sigma_{t-1,1}^{(n)}$, and one of the set $\rho_{t-2,2}^{(n)}$, each rule being in a different class, or

(d) includes a basic rule of the class $R(0)^{n-s} * R(\alpha)^{8}$ and one of the set $\rho_{t-2,2}^{(n)}$; here $s=t-1+k(n-t+1)$ and must be an integer $>t$.

The bound may be found as follows. We set

(4.1) $\quad \bar{\nu}_{a}=\bar{\nu}\left(\rho_{t, 2}^{(n)}\right)$,

(4.2) $\quad \bar{\nu}_{b}=\bar{\nu}\left(\sigma_{t, 1}^{(n)}\right)+\bar{\nu}\left(\rho_{t-1,2}^{(n)}\right)$,

(4.3) $\bar{\nu}_{c}=\bar{\nu}\left(\sigma_{t, 1}^{(n)}, \sigma_{t-1,1}^{(n)}\right)+\bar{\nu}\left(\rho_{t-2,2}^{(n)}\right)$,

$$
\begin{aligned}
\bar{\nabla}_{d} & =\propto, \quad k(n-t+1) \text { not a positive integer, } \\
& =\nu\left((R(0))^{m-s} *(R(\alpha))^{s}\right)+\bar{\nu}\left(\rho_{t-2,2}^{(n)}\right), \quad s=t-1+k(n-t+1) .
\end{aligned}
$$


These values $\bar{\nu}_{a}, \bar{\nu}_{b}, \bar{\nu}_{c}$, and $\bar{\nu}_{d}$ correspond to the possibilities $a, b$, $c$, and $d$ of Theorem 4.1. In terms of these quantities we write

$$
\bar{\nu}\left(S_{t+1}^{(n)}\right) \geqq \min \left(\bar{\nu}_{a}, \bar{\nu}_{b}, \bar{\nu}_{c}, \bar{\nu}_{d}\right) .
$$

This may be expressed explicitly in terms of $n$ and $t$ using the expressions given in Section 2 ; however, the expanded equation is unwieldy.

If $n \gg t$, we find

$$
\bar{\nu}\left(S_{t+1}^{(n)}\right)>2^{t}\left(\begin{array}{l}
n \\
t
\end{array}\right)+2^{t-1}\left(\begin{array}{c}
n \\
t-1
\end{array}\right)+(t-2) 2^{t-2}\left(\begin{array}{c}
n \\
t-2
\end{array}\right) .
$$

This may be compared with the one-parameter system $\bar{W}_{i+1}^{(n)}$, described in Sections 6 and 8 of Part III. There

$$
\nu\left(\bar{W}_{t+1}^{(n)}\right)=2^{t}\left(\begin{array}{l}
n \\
t
\end{array}\right)+2^{t-1}\left(\begin{array}{c}
n \\
t-1
\end{array}\right)+(t-1) 2^{t-2}\left(\begin{array}{c}
n \\
t-2
\end{array}\right)
$$

+ terms of lower degree in $n$.

The closeness of this lower bound (4.5) to the number of points used by previously published rules for integration over a hypercube is indicated in the table.

\begin{tabular}{|c|c|c|c|c|c|c|}
\hline$n$ & $\bar{\nu}_{a}$ & $\bar{\nu}_{b}$ & $\bar{\nu}_{c}$ & $\bar{\nu}_{d}$ & $\nabla\left(S_{5}{ }^{(n)}\right) \geqq$ & $R_{5}^{(n)} \dagger$ \\
\hline $\begin{array}{r}4 \\
6 \\
10 \\
15\end{array}$ & $\begin{array}{r}64 \\
384 \\
10,240 \\
87,360\end{array}$ & $\begin{array}{r}80 \\
448 \\
3,904 \\
33,760\end{array}$ & $\begin{array}{r}96 \\
344 \\
2,344 \\
26,320\end{array}$ & $\begin{array}{c}\propto \\
(360)^{*} \\
\propto \\
\propto\end{array}$ & $\begin{array}{r}64 \\
344 \\
2,344 \\
26,320\end{array}$ & $\begin{array}{r}169 \\
477 \\
2,645 \\
26,921\end{array}$ \\
\hline
\end{tabular}

\section{Numerical values for certain degree 9 rules}

$$
(t=4) \text {. }
$$

* This value applies in case $k=1 / 3$. In case $k=5 / 9$ this is $\propto$.

$\dagger$ These rules are $\bar{W}_{6}^{(n)}$ or $\bar{W}_{5}^{(n)}$ defined in Sections 6 and 8 of Part III. See Table 2 of Part III.

$\bar{\nu}_{a}, \bar{\nu}_{b}, \bar{\nu}_{c}$, and $\bar{\nu}_{d}$ are defined in equations (4.1) to (4.4) and the limit on $\bar{\nu}\left(S_{5}{ }^{(n)}\right)$ is (4.5). The table indicates that this bound is relatively close to the actual value of $\bar{\nu}\left(S_{5}{ }^{(n)}\right)$ for large $n$. It also gives information about the form of a minimal rule for large $n$. For $n=10$ and 15, the minimal rule must be of the form indicated in (c) of Theorem 4.1, since either choice (a) or choice (b) of Theorem 4.1 require more points than required by an existing rule.

Applied Mathematics Department

University of New Souih Wales

Kensington, N.S.W., Australia

1. J. N. Lyness, "Symmetric integration rules for hypercubes. I. Error coefficients," Math. Comp., v. 19, 1965, pp. 260-276.

2. J. N. LyNess, "Symmetric integration rules for hypercubes. II. Rule projection and rule extension," Math. Comp., v. 19, 1965, pp. 394-407.

3. J. N. LYNESS, "Symmetric integration rules for hypercubes. III. Construction of integration rules using null rules," Math. Comp., v. 19, 1965, pp. 625-637.

4. J. N. LYNESS, "Integration rules of hypercubic symmetry over a certain spherically symmetric space," Math. Comp., v. 19, 1965 pp. 471-476.

5. A. H. STROUd \& D. SECREST, "Approximate integration formulas for certain spherically symmetric regions," Math. Comp., v. 17, 1963, pp. 105-135. MR 28 *4677. 